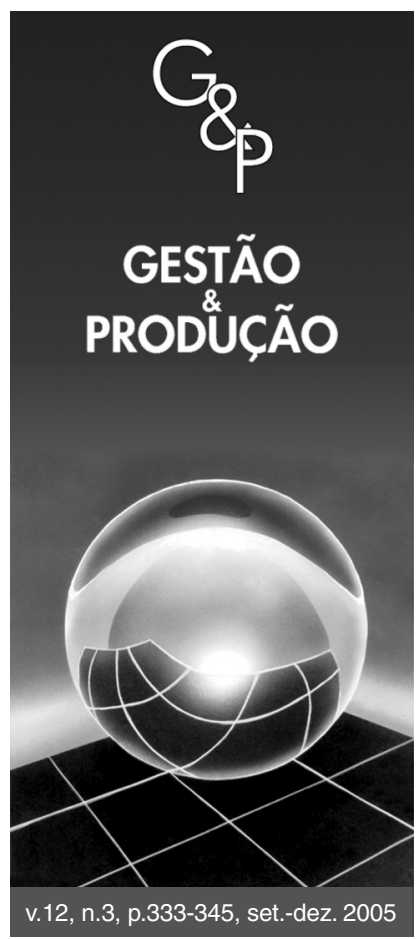

\title{
PARADIGMAS ESTRATÉGICOS DE GESTÃO DA MANUFATURA (PEGEMs): ELEMENTOS-CHAVE E MODELO CONCEITUAL
}

\author{
Moacir Godinho Filho \\ Flavio César Faria Fernandes
}

Departamento de Engenharia de Produção, Universidade Federal de São Carlos, UFSCar, Rod. Washington Luís, Km 235, C. P. 676, CEP 13565-905, São Carlos, SP, e-mail: moacir@dep.ufscar.br, dfcf@power.ufscar.br

Resumo

O presente artigo propõe um novo termo em Gestão da Produção: Paradigmas Estratégicos de Gestão da Manufatura (PEGEMs). Este novo conceito objetiva tratar de forma integrada e comparativa os paradigmas de gestão da manufatura surgidos ao longo do século XX. Para isto, além de sua conceituação, são identificados quatro elementos-chave comuns a todos os PEGEMs: direcionadores (condições do mercado que possibilitam ou requerem a implantação do PEGEM); objetivos de desempenho (os objetivos estratégicos da produção priorizados pelo PEGEM); princípios (as idéias que norteiam o PEGEM); e capacitadores (as ferramentas, tecnologias e métodos de cada PEGEM). As principais contribuições deste trabalho são: i) apresentar um modelo conceitual que relacione os PEGEMs aos objetivos estratégicos da produção; e ii) permitir comparações e análises dos paradigmas de manufatura, facilitando o estudo e aplicações práticas. O modelo proposto fornece um tratamento bastante pragmático das questões estratégicas no âmbito da Gestão da Produção.

Palavras-chave: paradigmas estratégicos de gestão da manufatura, manufatura em massa, manufatura enxuta, manufatura responsiva, customização em massa, manufatura ágil.

\section{Introdução}

Dentro da moderna literatura de Gestão da Produção, muitos são os paradigmas que se apresentam no intuito de ajudar as empresas na difícil tarefa de se manterem competitivas no mundo atual globalizado (Gunasekaran, 1999). Alguns exemplos são: Manufatura Enxuta, Manufatura Responsiva, Manufatura Ágil, World Class Manufacturing, Customização em Massa, dentre outros conceitos. Preferimos o termo Manufatura ao termo Produção por estar diretamente ligado à fabricação de bens e não contemplar a geração de serviços.

O presente trabalho tem como objetivo apresentar estes principais paradigmas atualmente existentes dentro da Gestão de Produção, tratando-os de uma forma integrada e comparativa. Para isso, um novo termo é proposto neste trabalho: Paradigmas Estratégicos de Gestão da
Manufatura (PEGEM). Este novo termo vem tratar de maneira conjunta e integrada os novos paradigmas dentro da moderna literatura de gestão da produção, por meio da criação de uma conceituação e de elementos-chave (direcionadores, objetivos de desempenho, princípios e capacitadores) comuns a todos estes paradigmas. A partir desta nova conceituação, podem-se diferenciar os paradigmas da manufatura de outros termos comumente encontrados na literatura, além de também permitir uma comparação entre os próprios paradigmas (à luz de seus elementos-chave), facilitando, assim, o estudo e a sua aplicabilidade. Os PEGEMs fornecem um aspecto estratégico à manufatura, uma vez que cada PEGEM é mais direcionado a determinados objetivos estratégicos da produção. Referente a esta questão, propomos um mo- 
delo que identifica os objetivos estratégicos da produção que cada PEGEM prioriza. Este modelo é posicionado em relação a outros dois modelos encontrados na literatura (Booth, 1996; Fernandes e MacCarthy, 1999).

Vergara (2000) classifica as pesquisas quanto aos fins (exploratória, descritiva, explicativa, metodológica, aplicada e intervencionista) e quanto aos meios (pesquisa de campo, pesquisa de laboratório, documental, bibliográfica, experimental, ex post facto, participante, pesquisa-ação e estudo de caso). Esta pesquisa se caracteriza quanto aos fins como exploratória, uma vez que busca sistematizar conhecimento que ainda não se encontra suficientemente integrado. Quanto aos meios, é uma pesquisa bibliográfica, porém composta de análises inspiradas na vivência dos autores em inúmeros estudos de caso já realizados.

O presente trabalho é um instrumento para uma maior aplicação prática dos paradigmas de manufatura, uma vez que trata os PEGEMs, muitas vezes considerados panacéias na literatura, de forma realista, ou seja, cada qual direcionado a certas situações específicas, com princípios, métodos e ferramentas destinadas a alcançar certos objetivos estratégicos da manufatura.

A estrutura do artigo é a seguinte: na seção 2, é definido o conceito de PEGEM, bem como são apresentados os principais PEGEMs; na seção 3, são apresentados os quatro elementos-chave de um PEGEM (os direcionadores, os objetivos de desempenho da produção, os princípios e os capacitadores) e mostradas comparações entre os mais importantes PEGEMs, no tocante a estes elementos-chave; na seção 4 , é proposto o modelo que identifica os objetivos estratégicos da manufatura priorizados por cada PEGEM; e na seção 5, são tecidas algumas considerações finais.

\section{Os paradigmas estratégicos de gestão da manufatura (PEGEMs): definição e elementos-chave}

Antes de passarmos à definição do que entendemos por PEGEM, vamos definir paradigma. Nos dicionários Michaelis, Silveira Bueno e Aurélio, paradigma é definido como padrão, modelo. Ao longo da história da manufatura, muitos paradigmas foram propostos para auxiliar na árdua tarefa de gerir a manufatura. Primeiramente surgiu a Manufatura em Massa, criada por Henry Ford no início do século XX. Este paradigma, aqui denominado Manufatura em Massa Atual (MMA), ainda é utilizado em empresas no mundo todo, porém com algumas diferenças em relação à sua forma original. Nos meados da década de 50, começa a surgir no Japão a Manufatura Enxuta (ME), a qual se consolida na década de 70. Mais recentemente, no final da década de 80 e início da década de 90 , foram desenvolvidos outros três importan- tes paradigmas: a competição baseada no tempo (também denominada de Manufatura Responsiva por autores como Kritchanchai e MacCarthy (1998) e Fernandes e MacCarthy (1999)), proposta primeiramente por Stalk e Hout (1990); a Customização em Massa (CM), que surgiu em 1987 com Stanley Davis em seu famoso livro "O futuro perfeito" (Davis, 1987); e a Manufatura Ágil (MA), que surgiu e foi popularizada em 1991 por um grupo de professores do Instituto Iaccoca da Universidade Lehigh, nos Estados Unidos, que publicaram, nesse mesmo ano, um relatório (Goldman et al., 1991), o qual trazia como a competitividade se desenvolveria nos próximos 20 anos, e propondo a MA como um novo paradigma destinado a este ambiente.

Acreditamos que todos estes paradigmas possam ser estudados de uma forma conjunta em prol de um maior entendimento, comparações e maior utilização prática. Para isso, propomos um novo conceito, que pretende abranger os paradigmas descritos: Paradigma Estratégico de Gestão da Manufatura (PEGEM). Conceituamos Paradigmas Estratégicos de Gestão da Manufatura (PEGEMs) como modelos/padrões estratégicos e integrados de gestão, direcionados a certas situações do mercado, que se propõem a auxiliar as empresas a alcançarem determinado(s) objetivo(s) de desempenho (daí o nome estratégicos). Estes paradigmas são compostos de uma série de princípios e capacitadores (daí a denominação gestão), os quais possibilitam que a empresa, a partir de sua função manufatura (daí a denominação manufatura), atinja tais objetivos, aumentando, desta forma, seu poder competitivo.

A partir desta explanação, denominamos PEGEM os seguintes paradigmas: Manufatura em Massa Atual (MMA); Manufatura Enxuta (ME); Manufatura Responsiva (MR); Customização em Massa (CM); e Manufatura Ágil (MA).

Vale ainda destacar que os PEGEMs acima estão em graus diferentes de comprovação, aplicação em situações reais e perspectivas futuras de aplicação: a MMA e a ME estão mais consolidadas, enquanto a MR, a CM e a MA têm uma taxa de crescimento bastante promissora em função dos avanços em tecnologia de informação e aumento da geração de riqueza em algumas partes do mundo.

A partir da definição anterior, podemos notar que um PEGEM é composto por quatro elementos-chave, os quais representam os pilares de um PEGEM (Figura 1). São eles:

(i) Direcionadores: Os direcionadores são as condições do mercado que possibilitam ou requerem ou facilitam a implantação de determinado PEGEM. A partir de uma revisão e análise crítica da literatura a respeito de todos os PEGEMs (Sharp et al., 1999; Yusuf et al., 1999, dentre outros), levantamos (Tabela 1) 
os principais direcionadores de cada um dos PEGEMs citados na seção anterior;

(ii) Objetivos de desempenho da produção: Cada PEGEM busca dotar a empresa de uma vantagem competitiva ao priorizar um objetivo de desempenho da produção, também chamado de objetivo estratégico da produção. Assim, um objetivo de desempenho da produção é um critério que posiciona estrategicamente a empresa em relação a seus concorrentes diretos. Enfatizando, cada PEGEM está relacionado a determinados objetivos de desempenho da produção, conforme discutido e proposto na seção 4. Dada a importância do modelo proposto na seção 4.3 , a seção 3 é dedicada a este tópico;

(iii) Princípios: Os princípios são as idéias que norteiam uma ação ou decisão; no caso, norteiam a empresa rumo aos objetivos de desempenho de um PEGEM. Os princípios representam o "o quê" deve ser atingido ou buscado; e

(iv) Capacitadores: Capacitadores são as ferramentas, tecnologias e métodos que devem ser implementados. Os capacitadores representam o "como" atingir um princípio.

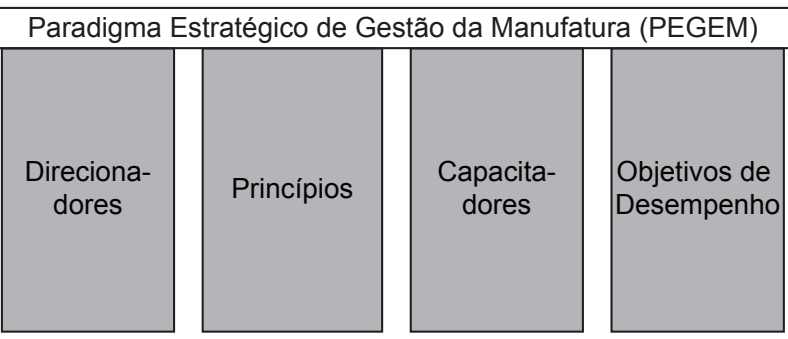

Figura 1. Os quatro elementos-chave de um Paradigma Estratégico de Gestão da Manufatura.
Vale ressaltar a relação entre esses 4 elementos-chave. A partir dos direcionadores surgem os objetivos de desempenho que a empresa deve priorizar. A partir daí, a empresa deve focar algumas idéias (princípios) relacionadas a esses objetivos. Já os capacitadores nos dizem como os princípios devem ser implementados de modo que os objetivos sejam atingidos.

Embora princípios e capacitadores sejam fáceis de definir, há uma intrincada relação entre eles e, assim, torna-se difícil separá-los de forma inquestionável: um princípio $X$, para se concretizar, necessita de um capacitador $\mathrm{Y}$ e este, por sua vez, requer um capacitador $\mathrm{Z}$, assim, para o capacitador $Z$, o $Y$ pode ser entendido como um princípio. Por exemplo, o princípio de focar o cliente sensível a baixos preços requer economia de escala e esta, por sua vez, pode requerer alta especialização de trabalho; assim, economia de escala pode ser entendida tanto como um capacitador quanto como um princípio. A esta relação denominamos cadeia de relacionamento princípio-capacitador. Esta cadeia é ilustrada na Figura 2. Este artigo não se preocupa com a discussão disso em detalhes, uma vez que ela não é necessária para a proposta do modelo (seção 4.3), que é o foco deste trabalho. O que é necessário é a identificação, para cada PEGEM, dos princípios/capacitadores mais enfatizados ou eventualmente exclusivos, a partir dos quais decorre(m) o(s) objetivo(s) de desempenho ganhador(es) de pedido. Isto é realizado na seção 4.3.

No final deste artigo, anexamos um check-list, baseado na literatura sobre PEGEMs, de princípios/capacitadores relacionados com os 5 PEGEMs.

\section{Os objetivos de desempenho da produção}

Nesta seção, são tratados os objetivos de desempenho da produção, também denominados objetivos estratégi-

Tabela 1. Os direcionadores dos PEGEMs.

\begin{tabular}{|c|c|c|c|c|}
\hline $\begin{array}{c}\text { Manufatura em } \\
\text { Massa Atual }\end{array}$ & Manufatura Enxuta & $\begin{array}{l}\text { Manufatura } \\
\text { Responsiva }\end{array}$ & $\begin{array}{c}\text { Customização em } \\
\text { Massa }\end{array}$ & Manufatura Ágil \\
\hline Mercado homogêneo & Mercado estável & $\begin{array}{l}\text { Mercado caracteriza- } \\
\text { do pela competição } \\
\text { baseada no tempo e na } \\
\text { diversidade de produtos. }\end{array}$ & $\begin{array}{l}\text { Customização em Massa } \\
\text { deve representar uma } \\
\text { característica diferencial, } \\
\text { como fonte de vanta- } \\
\text { gens competitivas no } \\
\text { mercado; além disso, } \\
\text { os produtos devem ser } \\
\text { customizáveis. }\end{array}$ & $\begin{array}{l}\text { Mercados totalmente } \\
\text { imprevisíveis marcados } \\
\text { por mudanças bruscas. }\end{array}$ \\
\hline $\begin{array}{l}\text { Clientes entendendo o } \\
\text { preço como o principal } \\
\text { diferencial competitivo }\end{array}$ & $\begin{array}{l}\text { Clientes desejando } \\
\text { preços, qualidade e } \\
\text { diferenciação. }\end{array}$ & $\begin{array}{l}\text { Clientes desejando ve- } \\
\text { locidade, pontualidade e } \\
\text { alta variedade, ou seja, } \\
\text { responsividade. }\end{array}$ & $\begin{array}{l}\text { Clientes desejando } \\
\text { customização. }\end{array}$ & $\begin{array}{l}\text { Clientes com desejos os } \\
\text { mais diversos possíveis } \\
\text { e mutáveis e necessidade } \\
\text { da empresa fazer frente } \\
\text { a este desafio. }\end{array}$ \\
\hline
\end{tabular}




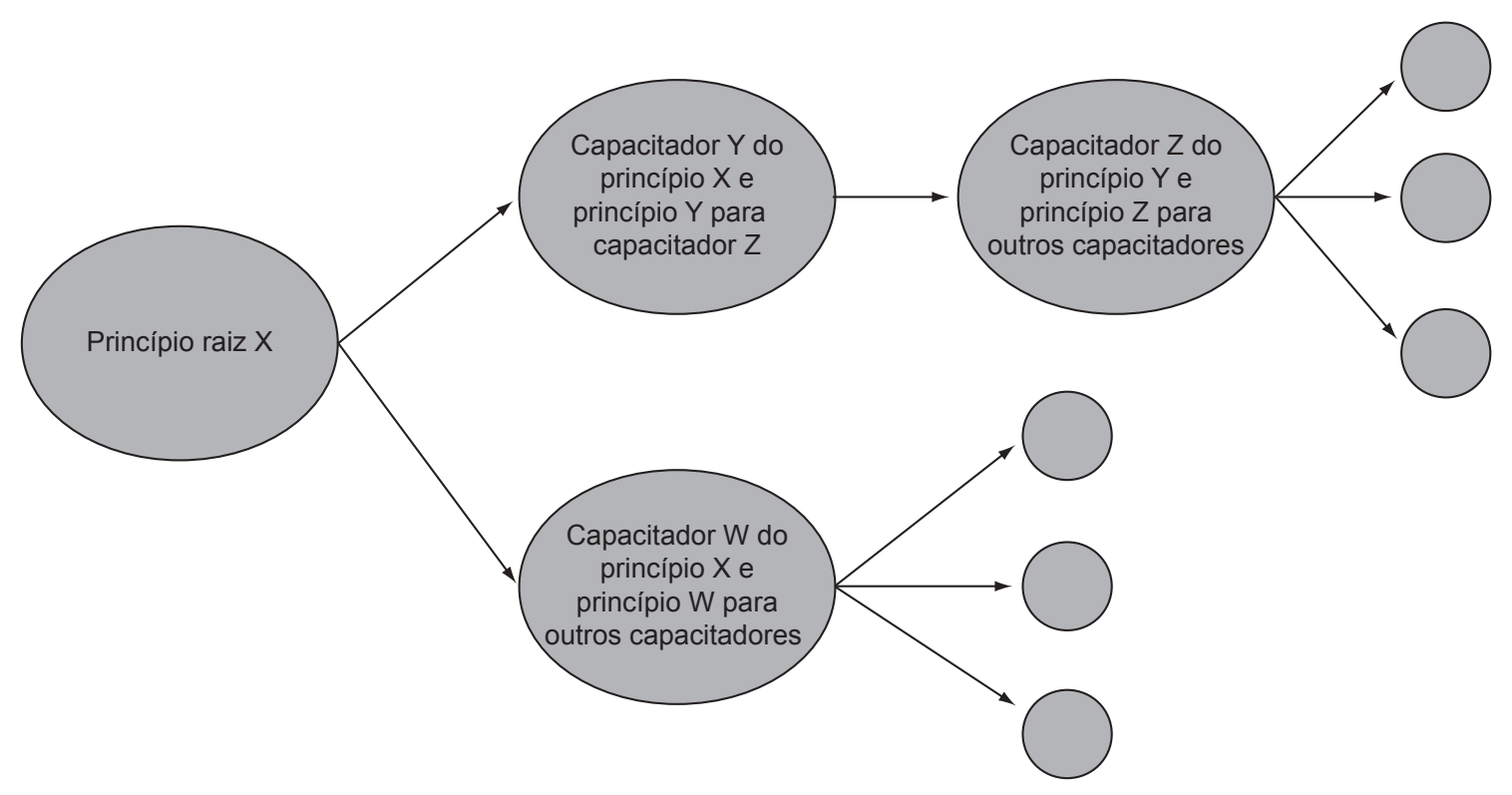

Figura 2. Exemplo de cadeia de relacionamento princípio-capacitador.

cos da produção. Estratégia de Produção é definida em Slack et al. (1997) como sendo o padrão global de decisões e ações, que define o papel, os objetivos, e as atividades da produção de forma que estes apóiem e contribuam para a estratégia de negócios da organização. Resumidamente, podemos dizer que uma estratégia de produção, primeiramente, determina a priorização dos objetivos de desempenho da produção. Baseadas nesta priorização de objetivos de desempenho, são estabelecidas as direções gerais para cada uma das principais áreas de decisão da produção (estas duas etapas são chamadas conteúdo da estratégia de produção nas palavras de Slack et al. (1997) e de Corrêa e Gianesi (1996)).

A partir destas definições entendemos que a função produção contribui para a realização da estratégia corporativa por meio da performance dos objetivos estratégicos da produção. Para Slack et al. (1997), estes objetivos são cinco: qualidade, rapidez (velocidade), pontualidade, flexibilidade e custo. Vamos considerar um número maior de objetivos uma vez que, com apenas cinco, não é possível caracterizar os cinco paradigmas do modelo proposto na seção 4.3. São eles:

- produtividade: habilidade do sistema de produção (SP) satisfazer a demanda dos consumidores em termos de baixo custo;

- qualidade 1: habilidade do SP satisfazer a demanda dos consumidores em termos de adequação ao uso;

- qualidade 2: habilidade do SP satisfazer a demanda em termos de desempenho ou conformidade a um preço aceitável (abordagem baseada no valor). Esta é a abordagem mais ampla e completa dentre as abordagens apontadas por Garvin (1992);
- flexibilidade 1: habilidade do SP responder a mudanças no mix de produtos dentro de uma gama limitada de opções, ou seja, o processo é capaz de fornecer diferenciação (pequena variedade de produtos alternativos bastante similares). Ela depende da obtenção de baixos tempos de set-up;

- flexibilidade 2: habilidade do SP responder a grandes mudanças no mix de produtos, ou seja, o processo é capaz de fornecer diversidade (grande variedade de produtos distintos). Ela depende da obtenção de baixos tempos de set-up, uso de equipamentos universais e versáteis e mão de obra versátil;

- rapidez (velocidade): habilidade do SP responder a mudanças no volume de produção. Ela depende da aceleração do processo para obter redução do tempo de ciclo;

- pontualidade: habilidade do SP satisfazer as necessidades dos consumidores em termos de prazo de entrega;

- customabilidade: habilidade do SP fornecer soluções individuais para clientes diferenciados dentro de um mix de produtos pré-estabelecido. A diferença entre customabilidade e o que denominamos flexibilidade 2 pode ser explicada por um exemplo encontrado em Duray et al. (2000). Para este autor, a flexibilidade $2 \mathrm{im}$ plica que o cliente tenha escolha, mas não necessariamente a especificação do produto, a qual se traduz pela customabilidade. Por exemplo: ter centenas de possibilidades de diferentes cafés da manhã indica a flexibilidade 2, já especificar exatamente a formulação do café da manhã indica customabilidade. Portanto customabilidade é bem mais amplo que a flexibilidade 2; e 
- adaptabilidade: habilidade do SP prosperar em um ambiente em constante mudança, caracterizado por inovações tecnológicas e necessidade incessante de lançamento de produtos inéditos. Para Goranson (1999), é a habilidade de lidar e responder a mudanças, sejam elas constantes ou inesperadas, acrescentando-se a isto a habilidade de saber tirar vantagens destas mudanças, entendendo-as como uma oportunidade. Esta última característica da adaptabilidade é defendida por autores como Goldman, et al. (1995) e Sharifi e Zhang (1999).

Dois conceitos importantes na elaboração do modelo proposto na seção 4.3 são objetivos ganhadores de pedido e objetivos qualificadores. Tais conceitos estabelecem um grau de priorização entre os diferentes objetivos de desempenho. Foram desenvolvidos por Hill (1989) (utilizados por diversos autores, dentre eles Slack et al., 1997 e Slack, 1993).

- Objetivos ganhadores de pedidos: são aqueles que contribuem diretamente para a realização de um negócio. Eles são vistos pelo cliente como fatores-chave da competitividade. Um aumento no desempenho em um objetivo ganhador de pedido resultará em mais negócios ou pelo menos no aumento da probabilidade da empresa conseguir mais pedidos; e

- Objetivos qualificadores: também são importantes para a empresa, mas não são os principais determinantes do sucesso competitivo. São os objetivos nos quais a empresa deve estar acima de um nível determinado para que ela seja inicialmente considerada pelos clientes como uma possível fornecedora. Abaixo deste nível crítico de desempenho, a empresa provavelmente não vai sequer entrar na concorrência. Acima deste nível a empresa passa a ser considerada pelos clientes, mas principalmente em termos de seus critérios ganhadores de pedido. Para um objetivo qualificador, qualquer melhora acima deste nível não acrescenta grandes benefícios competitivos.

\section{Proposta de um modelo que relaciona os PEGEMs aos objetivos estratégicos da produção}

Nesta seção é proposto o modelo que relaciona os PEGEMs e os objetivos estratégicos da produção. Além de utilizar a evolução histórica dos PEGEMs, bem como seus princípios e capacitadores exclusivos, o modelo proposto se baseia em mais dois pontos fundamentais: i) modelos anteriores que relacionam alguns PEGEMs e alguns objetivos estratégicos da produção; e ii) na existência de trade offs na manufatura. Portanto, para um melhor entendimento do modelo, focamos as duas próximas seções nestes dois temas, para, então, apresentarmos o modelo propriamente dito.

\subsection{Dois modelos que relacionam os objetivos estratégicos aos PEGEMs}

Apresentamos nesta seção dois modelos propostos na literatura que propõem uma relação entre alguns PEGEMs e alguns objetivos de desempenho da manufatura. O primeiro deles, proposto por Booth (1996), é mostrado na Figura 3. De acordo com este modelo, cada paradigma de gestão da manufatura tem enfoque alto, médio ou baixo em três objetivos da manufatura (custo, responsividade e flexibilidade). De acordo com este modelo, temos que, por exemplo, a produção baseada no tempo tem alto foco na responsividade (tempo) e no custo e médio na flexibilidade.

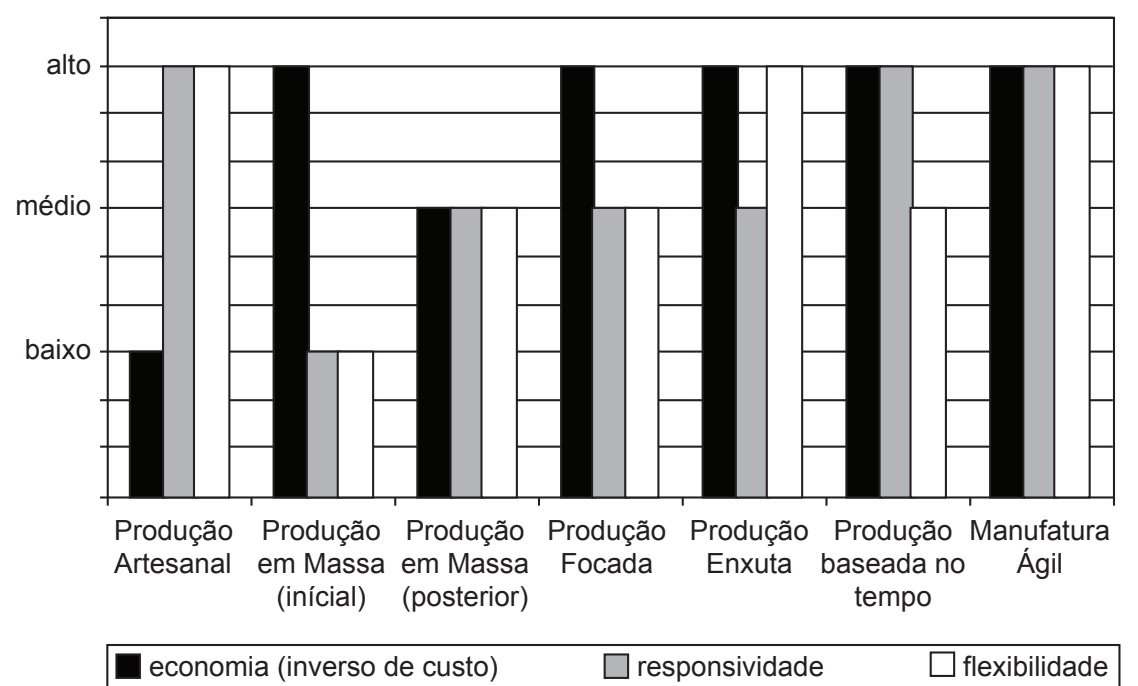

Figura 3. Primeiro modelo que relaciona alguns PEGEMs e alguns objetivos estratégicos da produção. Fonte: Booth (1996). 
Um segundo modelo que apresenta uma relação entre os Paradigmas Estratégicos de Gestão da Manufatura e os objetivos de desempenho da produção é o trabalho de Fernandes e MacCarthy (1999). Este modelo é apresentado na Figura 4. Podemos ver nesta figura que, à medida que se vai da Manufatura Repetitiva para a Manufatura Ágil, novos objetivos vão sendo levados em conta sem se desprezar os objetivos já incorporados. Por exemplo, a Manufatura Enxuta incorpora o objetivo qualidade e engloba o objetivo produtividade/custo da Manufatura Repetitiva e assim por diante.

\subsection{Os trade offs na manufatura}

A discussão entre os trade offs na manufatura é um dos pontos fundamentais para a proposição de nosso modelo, uma vez que é exatamente a existência de trade offs na manufatura que faz com que os PEGEMs não possam enfatizar da mesma forma todos os objetivos estratégicos ao mesmo tempo.

A idéia dos trade offs entre os objetivos da manufatura foi proposta pela primeira vez por Skinner (1969). Em sua opinião, os trade offs existem no projeto e operações dos sistemas de produção e este fato deve ser levado em

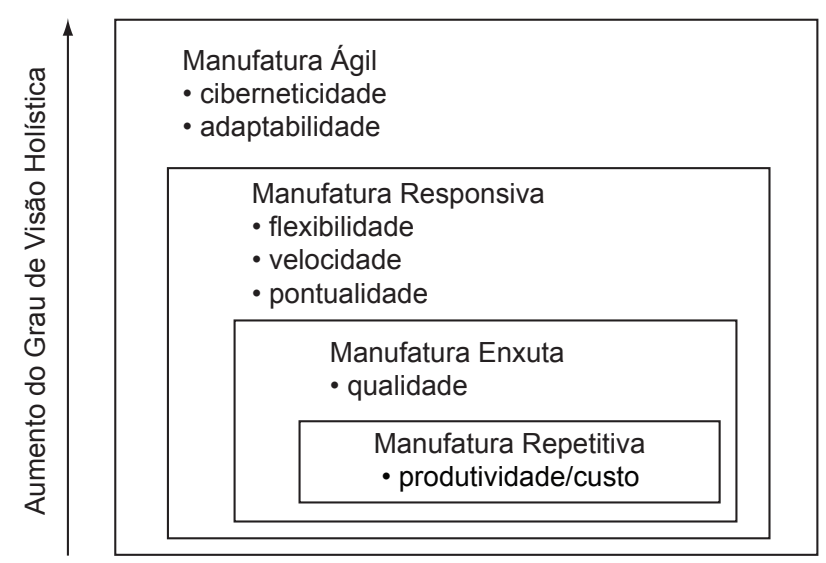

Figura 4. Segundo modelo que relaciona alguns PEGEMs e alguns objetivos estratégicos da produção. Fonte: Fernandes e MacCarthy (1999). consideração quando são projetados os sistemas de produção. Portanto, de acordo com este autor, as empresas devem concentrar seus esforços em um número reduzido de objetivos (de preferência somente um objetivo). Esta opinião é suportada por outros autores (Hayes e Wheelwright, 1984; Miller, 1983).

Após uma revisão bibliográfica dentro deste tema, identificamos na literatura de Gestão da Produção a coexistência de pelo menos quatro diferentes abordagens com relação aos trade offs na manufatura. Duas destas abordagens nos parecem bastante radicais (denominamos primeira e segunda) e outras duas mais realistas e, portanto, mais apropriadas (denominamos terceira e quarta).

A primeira abordagem defende claramente a existência de trade offs na manufatura, sendo, portanto, necessário que a empresa se foque em poucos ou somente um objetivo da manufatura. Esta abordagem é formada por autores como Miller (1983); Hayes e Wheelwright (1984).

Uma segunda abordagem, representada por muitos defensores da Manufatura Enxuta e da Manufatura de Classe Mundial, defende a não-existência de trade offs. O principal representante desta abordagem é Schonberger (1990), seguido por outros autores (Corbett e Wassenhove, 1993; Hill, 1988).

Uma terceira abordagem, não tão radical, entende que alguns trade offs existem e que outros não mais existem. Por outro lado, não há consenso entre quais trade offs pertencem a cada um desses dois grupos. Portanto, com base em New (1992), Khouja e Mehrez (1994) e Koste e Malhotra (2000), elaboramos a Tabela 2, que mostra os principais trade offs atualmente existentes.

Finalmente uma quarta vertente defende a existência dos trade offs, porém estes são dinâmicos e não estáticos, como eram considerados tempos atrás. Este dinamismo significa que medidas particulares, que variam de caso a caso, podem ser tomadas para que dois aspectos, aparentemente, inversamente proporcionais, possam ser melhorados, ao mesmo tempo, é claro, que se dá prioridade a um deles. Esta visão de trade offs dinâmicos entre os objetivos de desempenho da produção é mostrado em autores como Slack (1993), com sua idéia de "mover o

Tabela 2. Os trade offs atuais na manufatura.

\begin{tabular}{lccccccc}
\hline & Produtividade & Qualidade & Qualidade & Flexibilidade & Flexibilidade & Velocidade & Pontualidade \\
& & $\mathbf{1}$ & $\mathbf{2}$ & $\mathbf{1}$ & $\mathbf{2}$ & & \\
\hline Produtividade & X & SIM & SIM & SIM & SIM & - & - \\
Qualidade 1 & SIM & X & - & - & SIM & - & - \\
Qualidade 2 & SIM & - & X & - & SIM & SIM & SIM \\
Flexibilidade 1 & SIM & & - & X & - & - & - \\
Flexibilidade 2 & SIM & SIM & SIM & - & X & SIM & SIM \\
Velocidade & - & - & SIM & - & SIM & X & - \\
Pontualidade & - & - & SIM & - & SIM & - & X \\
\hline
\end{tabular}


pivô" e Hayes e Pisano (1996), com sua idéia do caminho da melhoria dinâmica. Outros autores dentro desta quarta categoria são Skinner (1992), Hayes e Pisano (1994) e Da Silveira e Slack (2001).

É claro que, mesmo com esta evolução na idéia dos trade offs na manufatura, acreditamos que eles existam, sendo, portanto, impossível para uma empresa ser a melhor em todos os aspectos ao mesmo tempo. Nas palavras de Correa (2001), "as prioridades competitivas da manufatura são estabelecidas porque um sistema de manufatura não pode ser o melhor em todos os aspectos ao mesmo tempo." Portanto estamos de acordo com as mais recentes (terceira e quarta) abordagens sobre trade offs, ou seja, eles ainda existem para alguns objetivos de desempenho (ver Tabela 2), sendo que a idéia dos trade offs dinâmicos é válida para muitos destes trade offs.

\subsection{0 modelo proposto e exemplos ilustrativos}

A idéia básica de nosso modelo é que novos PEGEMs surgiram em virtude do aparecimento de novas necessidades de mercado, as quais exigem, para serem satisfeitas, diferentes ênfases nos objetivos de desempenho. A Figura 5, proposta por Bolwijin e Kumpe (1990), mostra como as necessidades do mercado, e os objetivos de desempenho a eles relacionados, foram se alterando ao longo dos últimos 40 anos do século XX.

Outros suportes para a criação do nosso modelo foram:

- a literatura sobre Manufatura em Massa, Enxuta, Responsiva, Ágil e Customização em Massa, mais espe- cificamente sobre seus princípios (que algumas vezes podem ser entendidos como capacitadores, conforme o final da seção 2) mais enfatizados e eventualmente exclusivos. Os objetivos ganhadores de pedido de cada PEGEM surgem a partir deles (Tabela 3);

- nos trade offs entre os vários objetivos estratégicos da manufatura (mostrados na Tabela 2) - os trade offs da manufatura explicam as quedas de performance de alguns objetivos de desempenho, conforme os objetivos ganhadores de pedido vão se alterando; e

- nos dois modelos descritos anteriormente (Figuras 3 e 4), os quais serão, ainda nesta seção, confrontados com o modelo proposto.

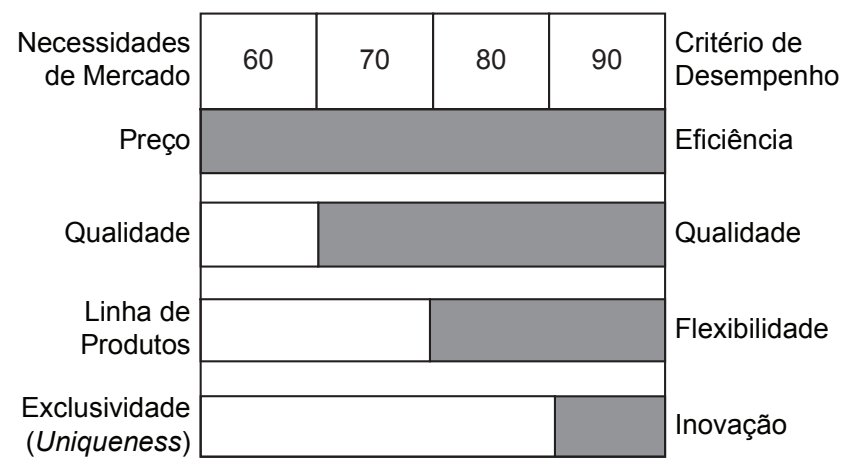

Figura 5. Evolução de necessidades de mercado e critérios de desempenho para empresas industriais de grande porte. Fonte: Bolwijn e Kumpe (1990).

Tabela 3. PEGEMs e seus princípios/capacitadores mais enfatizados ou eventualmente exclusivos e objetivo(s) estratégico(s) ganhador(es) de pedido relacionado(s).

\section{PEGEM Princípios/capacitadores mais enfatizados ou eventualmente exclusivos dos quais Objetivo(s) decorrem o(s) objetivo(s) estratégico(s) ganhador(es) de pedido \\ estratégico(s) \\ ganhador(es) \\ de pedido \\ relacionado(s)}

MMA Foco em clientes sensíveis aos baixos preços; foco na padronização do produto, sendo que alguma diferenciação é possível; foco na eficiência operacional/alta produtividade; alta especialização do trabalho.

ME Foco na qualidade; foco no fornecimento ao cliente de uma ampla diferenciação de produtos, com pouca diversidade; foco na identificação e eliminação de desperdícios; adoção da estratégia de controle da produção just in time, formada por vários princípios (produção puxada, etc...); autonomação.

MR Foco no atendimento de clientes que priorizam a diversidade de produtos, o tempo de resposta e o cumprimento de prazos; adoção de estratégia de controle da produção que foca a competição baseada no tempo num ambiente de alta variedade de produtos.

CM Foco no atendimento da demanda fragmentada para diferentes gostos e necessidades; redução no ciclo de desenvolvimento do produto e também no ciclo de vida dos produtos; participação do cliente ao longo das etapas do ciclo de vida dos produtos.

MA Foco na identificação de novas e inéditas oportunidades de negócios; gestão baseada em competências-chave; desenvolver habilidades para dominar mudanças e incertezas; empresa virtual.
Produtividade

Qualidade 2 e

Flexibilidade 1

Responsividade (Flexibilidade $2+$ Velocidade + Pontualidade)

Customabilidade

Adaptabilidade 
Um exercício que o leitor interessado poderia fazer é: identificar e colocar numa tabela T o grau de ênfase de um princípio/capacitador (do anexo 1) por cada um dos cinco PEGEMs. Desse exercício surgiriam os princípios/capacitadores (Tabela 3) mais enfatizados ou eventualmente exclusivos (grau de importância 5) para cada PEGEM, dos quais decorrem o(s) objetivo(s) estratégico(s) ganhador(es) de pedido. A tabela $\mathrm{T}$ completa seria imensa e suscetível a controvérsias, embora, com base na literatura dos últimos 10 anos, as células com valor 5 seriam inquestionáveis. Uma tabela T parcial é a Tabela 4, a qual mostra alguns exemplos ilustrativos.

Outro exercício que deixamos para o leitor é: baseado no penúltimo parágrafo da seção 2 , criar uma cadeia de relacionamento princípio/capacitador a partir de um princípio raiz, como sugerido pela Figura 2. Um check-list de princípios e capacitadores encontra-se no anexo.

A Figura 6 representa nosso modelo de relacionamento entre os PEGEMs e os objetivos estratégicos da manufatura.

A Manufatura em Massa Atual tem como objetivo ganhador de pedidos a produtividade; porém, também exis-

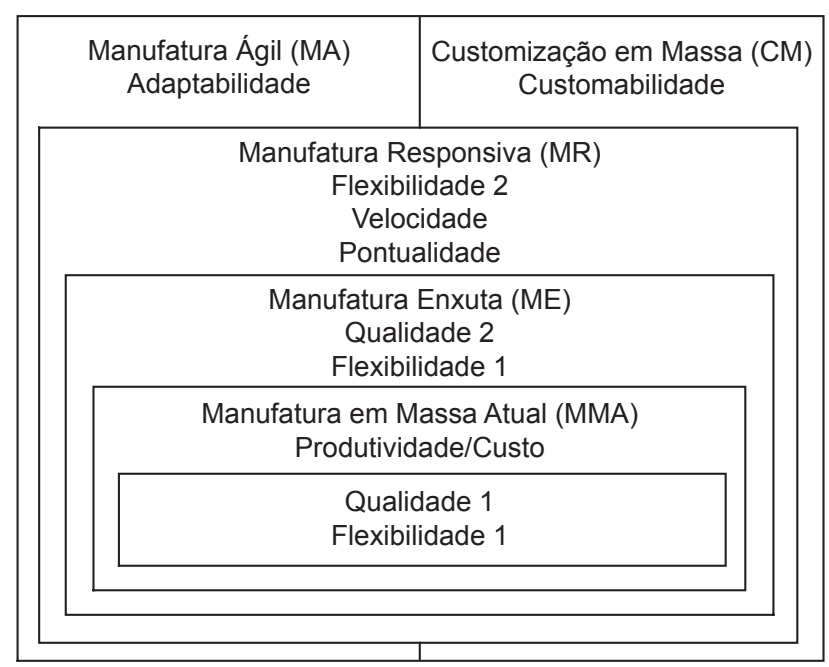

Figura 6. Modelo de relacionamento PEGEM - objetivos estratégicos da manufatura. tem preocupações com dois outros objetivos em nível de qualificadores: a qualidade 1 (o modelo $\mathrm{T}$ da Ford era adequado ao uso: robusto para enfrentar as trilhas de terra cheias de buracos e pedras) e a flexibilidade 1, uma vez que certa diferenciação é desejável na Manufatura em Massa Atual.

Um bom exemplo da Manufatura em Massa Atual é a empresa $\mathrm{X}$, que já está no mercado há quase 10 anos, A empresa $\mathrm{X}$ fabrica calçados femininos, principalmente, para as classes $\mathrm{C}$ e $\mathrm{D}$ (ganhador de pedido é o custo), possui pouca variedade de modelos, mas uma certa diferenciação tem sido necessária para se manter no mercado. O volume de produção é bastante grande e usa máquinas automatizadas nos processos de corte e montagem. Quanto à qualidade, a empresa considera adequado para o público alvo simplesmente substituir os calçados que o cliente julga defeituosos; a taxa de devolução é de $3 \%$ e, segundo cálculos da empresa, custaria mais investir nos processos e materiais para se conseguir diminuir essa taxa do que simplesmente fazer a substituição do item.

A Manufatura Enxuta surgiu da impossibilidade de, no Japão, em meados dos anos 1950, priorizar-se a produtividade, a qual requer geralmente grandes economias de escala - uma grande escala de produção para um único modelo seria impensável lá -, assim, a saída foi se diferenciar dos concorrentes em termos de flexibilidade $1 \mathrm{e}$ qualidade 2 . A produtividade é também um objetivo, pois ganhos neste objetivo levam a uma rentabilidade maior; mas não é possível colocá-la no mesmo nível de prioridade que a qualidade 2 e a flexibilidade 1 , já que existem trade offs (veja seção 4.2, Tabela 2) entre produtividade e qualidade 2 e entre produtividade e flexibilidade 1.

A fábrica de calçados masculinos $\mathrm{Y}$ é um exemplo de empresa que tem adotado a Manufatura Enxuta. A variedade de modelos é restrita em relação a outros fabricantes de calçados (por exemplo, a Democrata), mas a diferenciação é tão buscada quanto a qualidade.

A Manufatura Responsiva enfatiza a responsividade (flexibilidade $2+$ velocidade + pontualidade) e surgiu da necessidade de disponibilizar uma grande variedade de produtos, feitos sob encomenda, mas com um tempo de

Tabela 4. PEGEM x Grau de ênfase de alguns exemplos ilustrativos de princípios/capacitadores.

\begin{tabular}{|c|c|c|c|c|c|}
\hline Princípios/Capacitadores & MMA & ME & MR & $\mathbf{C M}$ & MA \\
\hline Alta Especialização do trabalho & 5 & 3 & 2 & 2 & 1 \\
\hline Autonomação & 1 & 5 & 2 & 2 & 2 \\
\hline $\begin{array}{l}\text { Estratégia de controle da produção que foca a competição baseada no tempo num } \\
\text { ambiente de alta variedade de produtos }\end{array}$ & 1 & 1 & 5 & 3 & 2 \\
\hline $\begin{array}{l}\text { Redução no ciclo de desenvolvimento do produto e também no ciclo de vida dos } \\
\text { produtos }\end{array}$ & 1 & 2 & 3 & 5 & 4 \\
\hline Empresa virtual & 1 & 1 & 1 & 2 & 5 \\
\hline
\end{tabular}

Legenda: grau 1 (ênfase baixa); grau 2 (ênfase baixa/média); grau 3 (ênfase média); grau 4 (ênfase média/alta); e grau 5 (ênfase alta). 
resposta (= leadtime de distribuição + leadtime de montagem + leadtime de fabricação de componentes (+ leadtime de suprimento, dependendo de a matéria-prima já existir ou não em estoque)) tão pequeno quanto possível, por estarmos no campo da competição baseada no tempo. Diante desta ênfase em flexibilidade 2, é impossível dar a mesma ênfase à qualidade 1 e 2 e à produtividade, uma vez que existem trade offs entre estes objetivos (ver Tabela 2).

A Kiddy, fabricante de calçados infantis, tem tentado atingir a Manufatura Responsiva a partir do primeiro semestre de 2004, uma vez que, em 2003, foi lançado um número grande de novos modelos (401). Houve também um esforço para a diminuição do tempo de resposta e aumento da rentabilidade, apesar de, em alguns meses, o volume total de produção ter diminuído. O Planejamento e Controle da Produção (PCP) passou a ter uma importância estratégica para a empresa, já que esta função é fundamental para se obter flexibilidade 2, velocidade e pontualidade concomitantemente. Houve também mudanças significativas no layout, devido a esse aumento de variedade e diminuição de volume de produção por modelo.

Com a globalização, existem grandes empresas comerciais que identificam oportunidades de negócio baseadas num grande volume de um item personalizado. Essas empresas contratam fabricantes que têm a customabilidade como ganhador de pedidos e, portanto, estes fabricantes adotam a Customização em Massa. Outros objetivos ainda têm de ser tratados, mas com menos ênfase (como qualificadores), uma vez que customabilidade enfatiza destacadamente a flexibilidade (leva a flexibilidade a um extremo) e este extremo, por sua vez, apresenta trade off com qualidade, produtividade e tempo de resposta (ver seção 4.2).

A Maide, fabricante de calçados femininos, produz $100 \%$ para exportação, atendendo a pedidos personalizados em grandes volumes, para o mercado europeu. A Tecnologia de Informação é amplamente empregada. A empresa chega a produzir mais de 1000 modelos diferentes de calçados em um ano. Está no mercado há mais de 10 anos e possui 2500 funcionários. O preço médio dos produtos da empresa é um pouco acima do preço dos concorrentes. Os equipamentos são flexíveis, há certa capacidade ociosa e muita utilização de subcontratação para acomodar as grandes variações na demanda. Há grande participação dos clientes nas etapas do ciclo de vida dos produtos.

A Manufatura Ágil surgiu em função das oportunidades de inovação no mercado, graças ao surgimento de novas tecnologias e clientes com disposição e disponibilidade de recursos para adquirir produtos inovadores. A empresa Ágil muda de segmentos de atuação de maneira freqüiente e, por isso, geralmente, é totalmente ou quase totalmente desverticalizada, e os parceiros que realizam a produção terceirizada são substituídos conforme o seg- mento de produtos que vão sendo projetados. Assim, a adaptabilidade é o objetivo ganhador de pedido e os demais são qualificadores, usando-se um raciocínio análogo ao que acontece com a Customização em Massa.

No Brasil, a Manufatura Ágil é difícil de ser encontrada. Conhecemos uma empresa, a empresa $\mathrm{Z}$, que leva a adaptabilidade a suas últimas conseqüências, a ponto de um empresário chamar o empresário da empresa $Z$ de "especulador da produção". A empresa Z já produziu: máquinas $\mathrm{CNC}$ para fabricação de sorvetes, quando houve o boom de sorvetes por quilo; máquinas de contar moedas, quando surgiu o uso de moedas em larga escala no Brasil; micro supermercado que é operado por um robô 24 horas por dia; rastreador de veículos por satélite; dispositivo que possibilita o motor a combustão usar álcool ou gasolina; dentre muitos outros produtos. A competência-chave da empresa, que é comum a todos estes produtos é o uso da microeletrônica. A fabricação dos componentes é sempre feita por meio de parcerias, as quais mudam conforme o(s) tipo(s) de produto(s) comercializado(s) no momento.

Finalizando a seção, confrontamos o modelo proposto com os dois modelos descritos anteriormente (respectivamente Figuras 3 e 4). O modelo de Fernandes e MacCarthy (1999) retrata a evolução histórica (representada pela Figura 5) das ênfases nos objetivos de desempenho de uma forma muito mais precisa que o modelo de Booth (1996). Já nosso modelo é uma evolução do modelo de Fernandes e MacCarthy (1999), pelos seguintes motivos:

- o termo MMA é menos sujeito à confusão do que manufatura repetitiva;

- explicita que na MMA há objetivos qualificadores (qualidade 1 e flexibilidade1);

- distingue dois tipos de flexibilidade (1 e 2);

- distingue dois tipos de qualidade (1 e 2);

- introduz o paradigma Customização em Massa; e

- retira a ciberneticidade como objetivo ganhador de pedido. Ciberneticidade é a habilidade dos gerentes de produto e produção usarem, de forma efetiva, a tecnologia da informação para auxiliar na satisfação de determinados objetivos estratégicos de desempenho. Porém entendemos que ele é um capacitador e não um objetivo propriamente dito, já que não está ligado diretamente às necessidades dos consumidores.

Confrontando, ainda, nosso modelo com o modelo de Fernandes e MacCarthy (1999), notamos que ambos mantêm a mesma idéia: o(s) objetivo(s) explicitado(s) logo abaixo do nome do PEGEM (ver Figura 6) são objetivos ganhadores de pedido e os objetivos que aparecem dentro dos retângulos internos ao PEGEM se tornam objetivos qualificadores. Por exemplo, no nosso modelo, 
responsividade (flexibilidade $2+$ velocidade + pontualidade) é o objetivo ganhador de pedidos para a Manufatura Responsiva, enquanto que os objetivos qualidade 1 e 2 , flexibilidade 1 e produtividade/custo são os qualificadores deste PEGEM.

\section{Conclusões}

Este artigo apresenta a evolução e um melhor entendimento dos principais paradigmas de gestão da manufatura, surgidos no século XX. A partir desta evolução foi criado um conceito dentro da Gestão da Produção, o conceito de Paradigmas Estratégicos de Gestão da Manufatura (PEGEMs). A criação deste novo conceito, bem como a identificação de seus elementos-chave, cumpre alguns objetivos importantes:

- fornece um tratamento bastante pragmático das questões estratégicas no âmbito da Gestão da Produção;
- uniformiza conceitos dentro da Gestão da Produção e possibilita a comparação dos PEGEMs a outros termos bastante utilizados na Gestão da Produção;

- fornece base para um maior conhecimento, divulgação e aprofundamento de pesquisas relativas ao assunto; e

- possibilita o aparecimento de análises comparativas entre os PEGEMs.

Este trabalho apresenta também um modelo que relaciona os PEGEMs aos objetivos estratégicos da produção. Este modelo é mais completo do que outros modelos existentes na literatura. Ele mostra que existe um relacionamento claro entre os PEGEMs e os objetivos estratégicos da manufatura. Este modelo cumpre o objetivo de mostrar que cada empresa deve buscar o PEGEM mais adequado a seus objetivos estratégicos, e que não existe nenhum paradigma (por exemplo, a Manufatura Ágil) que seja panacéia para todas as situações.

\section{Referências Bibliográficas}

BOOTH, R. Agile Manufacturing. Engineering Management Journal, v. 6, n. 2, p. 105-112, April 1996.

BOLWIJN, P. T.; KUMPE, T. Manufacturing in the 1990s - productivity, flexibility and innovation. Long Range Planning, v. 23, n. 4, p. 44-57, 1990.

CORBETT, C.; WASSENHOVE, L. Trade offs? What trade offs? Competence and competiveness in manufacturing strategy. California Management Review, v. 35, n. 4, p. 107-122, 1993.

CORRÊA, H. L. Agile Manufacturing as the $21^{\text {st }}$ Century Strategy for Improving Manufacturing Competitiveness, in: GUNASEKARAN, A. (editor): Agile Manufacturing: the $21^{\text {st }}$ Century Competitive Strategy, Elsevier, 2001.

CORRÊA, H. L.; GIANESI, I. G. N. Just in Time, MRP II e OPT - Um enfoque estratégico. Ed. Atlas, $2^{a}$ Edição, 1996.

CROSBY, P. B. Quality is free. Mc Graw Hill, New York, 1979.

DA SILVEIRA, G; SLACK, N. Exploring the trade off concept. International Journal of Operations and Production Management, v. 21, n. 7, p. 949-964, 2001.

DAVIS, S. M. Future perfect. Mass: Addison Wesley, 1987.

DURAY, R.;WARD, P.T.; MILLIGAN, G.W.; BERRY, W. L. Approaches to mass customization: configurations and empirical validation. Journal of Operations Management, v. 18, n. 6, p. 605-625, 2000.

FERNANDES, F. C. F.; MACCARTHY, B. L. Production Planning and Control: the gap between theory and prac- tice in the light of modern manufacturing concepts. $15^{\text {th }}$ International Conference on CAD/CAM, Robotics \& Factories of the Future (CARS \& FOF'99). Proceedings..., Aguas de Lindóia-Brazil, v. 1, p. MF2-1 - MF2-6, August 1999.

GARVIN, D. Gerenciando a Qualidade. Rio de Janeiro: Ed. Qualitymark, 1992.

GOLDMAN, S. L.; NAGEL, R. N.; PREISS, K. Agile Competitors and Virtual Organizations, Van Nostrand Reinhold, New York, 1995.

GOLDMAN, S. L.; NAGEL, R. N.; PREISS, K.; DOVE, R. Iacocca Institute: $21^{\text {st }}$ Century Manufacturing Enterprise Strategy, An industrial Led View, v. 1-2. Iacocca Institute, Bethlehem, PA, 1991.

GORANSON, H. T. The Agile Virtual Enterprise - Cases, Metrics, Tools. Quorum Books, Westport, Connecticut, London, 1999.

GUNASEKARAN, A. Agile manufacturing: A framework for research and development. International Journal of Production Economics, v. 62, n. 1-2, p. 87-105, 1999.

HAYES, R. H.; PISANO, G. Beyond world class - the new manufacturing strategy. Harvard Business Review, v. 72, n. 1, p. 77-86, 1994.

HAYES, R. H.; PISANO, G. Manufacturing Strategy: at the intersection of two paradigm shifts. Production and Operations Management, v. 5, n. 1, p. 25-41, 1996.

HAYES, R. H.; WHEELWRIGHT, S. C. Restoring Our Competitive Edge: Competing Through Manufacturing. Wiley, New York, 1984. 
HILL, C. W. Differentiation versus low cost or differentiation and low cost: a contingency framework. Academic Management Review, v. 13, n. 3, p. 401-412, 1988.

HILL, T. Manufacturing Strategies - Text and cases, Irwin, Richard Irwin, USA, Boston, Massachussets, 1989.

KHOUJA, M.; MEHREZ, A. Economic Production Lot Size Model with Vanable Production Rate and Imperfect Quality, Journal of the Operational Research Society, v. 45, n. 12, p. 1405-1417, 1994.

KOSTE, L. L.; MALHOTRA, M. K. Trade offs among the elements of flexibility: a comparison from the automotive industry. OMEGA - The International Journal of Management Science, v. 28, p. 693-710, 2000.

KRITCHANCHAI, D.; MACCARTHY, B. L. Responsiveness and strategy in manufacturing. Proceedings of the Workshop Responsiveness in Manufacturing, digest n 98/213, IEE, London, 1998.

MACCARTHY, B. L.; FERNANDES, F. C. F. A multi-dimensional classification of production systems for the design and selection of production planning and control systems. Production Planning \& Control, v. 11, n. 5, p. 481-496, 2000.

MILLER, S. S. Make your plant manager's job manageable. Harvard Business Review, v. 61, n. 1, p. 69-74, 1983.

NEW, C. World-Class Manufacturing versus Strategic trade offs. International Journal of Operations and Production Management, v. 12, n. 6, p. 19-31, 1992.

SHARIFI, H.; ZHANG, Z. A methodology for achieving agility in manufacturing organizations: An Introduction.
International Journal of Production Economics, v. 62, n. 1-2, p. 7-22, 1999.

SHARP, J. M.; IRANI, Z.; DESAI, S. Working towards agile manufacturing in the UK industry. International Journal of Production Economics, v. 62, n. 1-2, p. 155-169, 1999.

SCHONBERGER, R. J. Building a Chain of Custumers: Linking Business Functions to Create the World Class Company. Free Press, New York, 1990.

SKINNER, W. Manufacturing - missing link in corporate strategy. Harvard Business Review, v. 47, n. 3, p. 136-145, 1969.

SKINNER, W. Missing the links in manufacturing strategy. In: VOSS, C.A. (ed.). Manufacturing Strategy Process and content. Chapman and Hall, London, p. 13-25, 1992.

SLACK, N., CHAMBERS, S., HARLAND, C., HARRISON, A.; JOHnStON, R. Administração da Produção. Ed. Atlas, Brasil SP, 1997.

SLACK, N. Vantagem Competitiva em Manufatura. Ed. Atlas, São Paulo, 1993.

STALK, G. Jr.; HOUT, T. M. Competing against time. Free Press, USA, 1990.

VERGARA, S. C. Projetos e relatórios de pesquisa em administração ( $3^{\text {a }}$ edição). São Paulo: Atlas, 2000.

YUSUF, Y. Y.; SARHADI, M.; GUNASEKARAN, A. Agile manufacturing: The drivers, concepts and attributes. International Journal of Production Economics, v. 62, n. 1-2, 1999.

\section{Anexo: Check-list, baseado na literatura, de princípios e capacitadores relacionados com os 5 PEGEMs}

- Foco em clientes sensíveis aos baixos preços;

- Foco na padronização do produto, sendo que alguma diferenciação é possível;

- Foco na eficiência operacional/alta produtividade;

- Alta especialização do trabalho;

- Foco na qualidade;

- Foco no fornecimento ao cliente de uma ampla diferenciação de produtos, com pouca diversidade;

- Foco na identificação e eliminação de desperdícios;

- Adoção da estratégia de controle da produção just in time, formada por vários princípios (produção puxada, etc.);

- Autonomação;

- Foco no atendimento de clientes que priorizam a diversidade de produtos, o tempo de resposta e o cumprimento de prazos;
- Adoção de estratégia de controle da produção que foca a competição baseada no tempo num ambiente de alta variedade de produtos;

- Foco no atendimento da demanda fragmentada para diferentes gostos e necessidades;

- Redução no ciclo de desenvolvimento do produto e também no ciclo de vida dos produtos;

- Participação do cliente ao longo das etapas do ciclo de vida dos produtos;

- Foco na identificação de novas e inéditas oportunidades de negócios;

- Gestão baseada em competências-chave;

- Desenvolver habilidades para dominar mudanças e incertezas;

- Empresa virtual;

- Simplificar ao máximo o fluxo de materiais; 
- Determinar o que é valor para o cliente;

- Identificação da cadeia de valor;

- Busca do zero defeito (perfeição);

- Qualidade Seis sigma;

- Gerenciamento Visual;

- $5 \mathrm{~S}$;

- Estabelecer o ritmo da inovação no seu setor industrial;

- Sistema integrado de trabalho em toda a cadeia e estruturado para a eliminação de tempos desnecessários;

- Sincronização da programação da produção e das capacidades na cadeia de suprimentos;

- Área de projetos voltada para reduzir a complexidade dos produtos e facilitar a manufatura;

- Cadeia de suprimentos preparada para a customização em massa;

- Flexibilizar o processo produtivo e o projeto de produtos;

- Criar e compartilhar o conhecimento (gestão baseada no conhecimento) e informações;

- Preços (um pouco) acima da média para compensar perda de eficiência;

- Utilização de módulos padrões;

- Integração na cadeia;

- Cooperação interna e externa para o aumento da competitividade;

- Desenvolver o espírito de pró atividade e empreendedorismo nas pessoas;

- Redução dos ciclos de vida dos processos e "da empresa";

- Economia de escala;

- Economia de escopo;

- Uso intensivo de máquinas especializadas;

- Adoção de características do sistema de produção de acordo com MacCarthy e Fernandes (2000), tais como: nível de repetitividade, estratégias de atendimento da demanda (make-to-stock, assembly-to-order, make-toorder, resources-to-order, engineering-to-order), tipos de lay-out e nível de automação compatíveis com a lógica do PEGEM;

- Adoção de sistemas de controle da produção (kanban, CONWIP, MRP, PBC, etc.) compatíveis com a lógica do PEGEM;
- Uso intensivo de peças intercambiáveis;

- Uso da linha de montagem cadenciada mecanicamente;

- Utilização de estudos de tempos e métodos e análise detalhada do processo para a sua padronização;

- Mapeamento do Fluxo de Valor;

- Melhoria na relação cliente-fornecedor;

- Redução do número de fornecedores;

- Recebimento Just-in-Time;

- Trabalho em Fluxo Contínuo;

- Redução de set-up e conseqüentemente do tamanho de lote;

- Trabalhar de acordo com o takt time;

- Manutenção Produtiva Total (TPM);

- Kaizen;

- Poka Yoke;

- Empowerment;

- Trabalho em equipes e participação;

- Comprometimento da gerência;

- Trabalhador multi-habilitado;

- Rodízio de funções;

- Treinamento e educação de pessoal;

- Adotar medidas de desempenho (Balanced Scorecard,...) e sistemas de custeio da produção (Sistema $\mathrm{ABC}, \ldots$...) compatíveis com a lógica do PEGEM;

- Gráficos de controle visuais;

- DFMA (design for manufacturing and assembly);

- Engenharia simultânea;

- CAD e CAM;

- Existência de uma rede de fornecedores confiável com relação aos prazos;

- Tecnologias e sistemas de informação voltados para integração interna e melhoria da eficiência no quesito tempo;

- Tecnologia e sistemas de informação voltados para a integração interna (ERP, intranet, etc.) e externa (EDI, etc.);

- Production Flow Analyses;

- Projeto do produto voltado à customização;

- Ciberneticidade; e

- Manufatura virtual. 


\section{STRATEGIC MANUFACTURING MANAGEMENT PARADIGMS (SMMPs): KEY ELEMENTS AND CONCEPTUAL MODEL}

\section{Abstract}

This paper proposes a new concept in Production Management: Strategic Manufacturing Management Paradigms (SMMPs). This new concept involves an integrated and comparative approach to the manufacturing management paradigms that emerged along the $20^{\text {th }}$ century. To this end, four key elements that are common to all SMMPs are identified: guiders (market conditions that allow for or require SMMP implementation); performance goals (the strategic manufacturing goals prioritized by the SMMPs); principles (the basic ideas that guide the SMMPs); and enablers (the SMMPs' tools, technologies and methods). The main contributions of this paper are: $i$ ) the presentation of a conceptual model that associates the SMMPs to strategic manufacturing goals; and ii) to allow for comparisons and analyses of manufacturing paradigms, facilitating their study and practical application. The proposed model takes a highly pragmatic approach to strategic Production/Operations Management issues.

Keywords: strategic manufacturing management paradigms, mass production, lean manufacturing, responsive manufacturing, mass customization, agile manufacturing. 